\title{
Approximation and the $n$-Berezin transform of operators on the Bergman space
}

\author{
Daniel Suárez
}

\begin{abstract}
To any bounded operator $S$ on the Bergman space $L_{a}^{2}$ we associate a sequence of linear transforms $B_{n}(S) \in L^{\infty}(D)$, where $n \geq 0$, and prove that the Toeplitz operators $T_{B_{n}(S)}$ tend to $S$ for some especial classes of operators $S$. In particular, this holds for every radial operator in the Toeplitz algebra. Finally, we show that the inclusion of the Toeplitz algebra into the essential commutant of the Bergman shift is proper.
\end{abstract}

\section{Introduction and preliminaries}

Let $\mathfrak{L}\left(L_{a}^{2}\right)$ be the algebra of bounded operators on the Bergman space $L_{a}^{2}=L_{a}^{2}(D, d A)$, and $L^{\infty}=L^{\infty}(D, d A)$, where $D$ is the unit disk and $d A$ is the normalized Lebesgue measure. The Toeplitz operator with symbol $a \in L^{\infty}$ is defined by $T_{a} f=P(a f)$, where $f \in L_{a}^{2}$ and $P$ is the orthogonal projection from $L^{2}(d A)$ onto $L_{a}^{2}$. The Toeplitz algebra $\mathfrak{T}\left(L^{\infty}\right)$ is the closed subalgebra of $\mathfrak{L}\left(L_{a}^{2}\right)$ generated by $\left\{T_{a}: a \in L^{\infty}\right\}$.

In [10] we use a sequence of linear transforms (the $n$-Berezin transforms) $B_{n}: \mathfrak{L}\left(L_{a}^{2}\right) \rightarrow L^{\infty}$, $n \geq 0$, to study some problems of approximation and abelianization of algebras generated by Toeplitz operators. Many authors have established the utility of the 0-Berezin transform of an operator $S$ as a tool to study some of its properties, especially when $S$ is a Toeplitz operator or belongs to $\mathfrak{T}\left(L^{\infty}\right)$ (see, for instance [2] and [12]). The 0-Berezin transform of

\footnotetext{
${ }^{0} 2000$ Mathematics Subject Classification: primary 32A36, secondary 47B35. Key words: Bergman space, Toeplitz operators, Berezin transforms.
} 
$S \in \mathfrak{L}\left(L_{a}^{2}\right)$ is usually called 'the Berezin transform of $S$ ' and denoted $\tilde{S}$. Since this paper deals with an indexed family of such transforms, we will not adopt that notation here.

Our main purpose is to show that some bounded operators $S$ are the norm limit of $T_{B_{n}(S)}$. Clearly, the above convergence implies that $S \in \mathfrak{T}\left(L^{\infty}\right)$, a necessary condition that we suspect is also sufficient. We give next a brief overview of the content of the paper. The main results are Theorems 2.4 and 3.3; most of the other results are either preparatory lemmas or corollaries of these theorems.

In this section we define $B_{n}(S)$, fix notation, state some known results and give a summary of the algebraic properties of $B_{n}$ that will be used later. All the results about $B_{n}$ listed here are proved in [10]. Section 2 provides criteria on an operator $S$ for the convergence of $T_{B_{n}(S)}$ to $S$ in the weak and the norm operator topologies. Theorem 2.4 gives a sufficient condition for norm convergence. As applications, we obtain that norm convergence holds for a Toeplitz operator $T_{\mu}$, where $|\mu|$ is a Carleson measure on Bergman spaces, and give characterizations of these operators and of Toeplitz operators with bounded symbol in terms of their $n$-Berezin transforms. In Section 3 we deal with radial operators (i.e.: diagonal operators with respect to $\left\{z^{n}\right\}_{n \geq 0}$ ), and show that every radial operator $S$ in the Toeplitz algebra is the norm limit of $T_{B_{n}(S)}$. In the process we obtain a new formula for $T_{B_{n}(S)}$ when $S$ is radial that could have further applications. We also show that a radial operator $S$ is compact if and only if $S \in \mathfrak{T}\left(L_{a}^{2}\right)$ and $B_{0}(S) \equiv 0$ on $\partial D$. The last section is devoted to answer a question of Englis [5], by showing that the inclusion of $\mathfrak{T}\left(L^{\infty}\right)$ in the essential commutant of $T_{z}$ is proper. One of the main ingredients of this proof is Theorem 3.3 from the previous section. We finish the paper posing some open problems.

If $z \in D$, let $\varphi_{z}(w)=(z-w) /(1-\bar{z} w)$ be the conformal map of $D$ that interchanges 0 and $z$. The pseudo-hyperbolic metric on $D$ is defined as $\rho(z, w)=\left|\varphi_{z}(w)\right|$. The 'change of variables' operator is $U_{z} f=\left(f \circ \varphi_{z}\right) \varphi_{z}^{\prime}\left(z \in D\right.$ and $\left.f \in L_{a}^{2}\right)$. It is easy to see that $U_{z}$ is unitary, self-adjoint, and that if $a_{1}, \ldots, a_{n} \in L^{\infty}$,

$$
U_{z} T_{a_{1}} \ldots T_{a_{1}} U_{z}=T_{a_{1} \circ \varphi_{z}} \ldots T_{a_{1} \circ \varphi_{z}}
$$

For $S \in \mathfrak{L}\left(L_{a}^{2}\right)$ we will write $S_{z} \stackrel{\text { def }}{=} U_{z} S U_{z}$. For a nonnegative integer $n$ and $z \in D$, denote

$$
K_{z}^{(n)}(\omega)=\frac{1}{(1-\bar{z} \omega)^{2+n}}(\omega \in D) .
$$

The $n$-Berezin transform of an operator $S \in \mathfrak{L}\left(L_{a}^{2}\right)$ is

$$
B_{n}(S)(z) \stackrel{\text { def }}{=}(n+1)\left(1-|z|^{2}\right)^{2+n} \sum_{j=0}^{n}\left(\begin{array}{l}
n \\
j
\end{array}\right)(-1)^{j}\left\langle S\left(\omega^{j} K_{z}^{(n)}\right), \omega^{j} K_{z}^{(n)}\right\rangle
$$


where $\langle$,$\rangle denotes the usual integral pairing and \left(\begin{array}{l}n \\ j\end{array}\right)=n ! /(n-j)$ ! j!. When $S=T_{a}$, with $a \in L^{\infty}(D)$, a simple calculation involving the equality $\sum_{j=0}^{n}\left(\begin{array}{l}n \\ j\end{array}\right)(-1)^{j}|\omega|^{2 j}=\left(1-|\omega|^{2}\right)^{n}$ and the change of variables $\omega=\varphi_{z}(\zeta)$ gives

$$
B_{n}(a)(z) \stackrel{\text { def }}{=} B_{n}\left(T_{a}\right)(z)=\int_{D} a\left(\varphi_{z}(\zeta)\right)(n+1)\left(1-|\zeta|^{2}\right)^{n} d A(\zeta) .
$$

This is the usual Berezin transform of the function $a$ with respect to the weighted Bergman space $L_{a}^{2}\left(d A_{n}\right)$, where $d A_{n}(\zeta)=(n+1)\left(1-|\zeta|^{2}\right)^{n} d A(\zeta)$, (see [1] and [7], Ch. 2). Since $d A_{n}(\zeta)$ is a probability measure, $B_{n}(a)(z)$ is an average of $a$ satisfying $\left\|B_{n}(a)\right\|_{\infty} \leq\|a\|_{\infty}$ for all $a \in L^{\infty}(D)$. For a general $S \in \mathfrak{L}\left(L_{a}^{2}\right)$ we only have

$$
\left\|B_{n}(S)\right\|_{\infty} \leq(n+1) 2^{n}\|S\| .
$$

Observe that this gives two different bounds for $\left\|B_{n}\left(T_{a}\right)\right\|_{\infty}$ that are independent of each other. In [10], Coro. 4.6, it is proved that

$$
B_{n}(S) \in \mathcal{A}=\left\{a \in L^{\infty}: a \text { is uniformly continuous with respect to } \rho\right\}
$$

for every $S \in \mathfrak{L}\left(L_{a}^{2}\right)$. It follows from the formula of $B_{n}(a)$ that $\left\|B_{n}(a)-a\right\|_{\infty} \rightarrow 0$ when $n \rightarrow \infty$ for every $a \in \mathcal{A}$. That is, the sequence $\left\{B_{n}\right\}$ works as an approximate identity for $\mathcal{A}$. In particular, $\lim \left\|T_{B_{n}(a)}-T_{a}\right\|=0$ for $a \in \mathcal{A}$. The next two properties are Corollary 2.7 and Lemma 2.2 of [10], respectively.

$$
\begin{gathered}
\left(B_{n} B_{k}\right)(S)=\left(B_{k} B_{n}\right)(S) \text { for every } n, k \geq 0 \text { and } S \in \mathfrak{L}\left(L_{a}^{2}\right) . \\
B_{n}\left(S_{z}\right)=B_{n}(S) \circ \varphi_{z} \text { for every } n \geq 0, S \in \mathfrak{L}\left(L_{a}^{2}\right) \text { and } z \in D .
\end{gathered}
$$

In particular, if we take $S=T_{a}$, with $a \in L^{\infty}$, (1.4) tells us that $B_{n}\left(a \circ \varphi_{z}\right)=B_{n}(a) \circ \varphi_{z}$. Also, suppose that $k$ is fixed in (1.3). Then $B_{k}(S) \in \mathcal{A}$ and the previous comments say that $B_{k}\left(B_{n}(S)\right)=B_{n}\left(B_{k}(S)\right) \rightarrow B_{k}(S)$ uniformly when $n \rightarrow \infty$. This observation will be at the core of some of our results.

\section{A criterion for approximation}

If $S_{k}, S \in \mathfrak{L}\left(L_{a}^{2}\right)$, for $k \geq 0$ integer, by $S_{k} \stackrel{\text { wOT }}{\rightarrow} S$ we mean that the sequence $S_{k}$ tends to $S$ in the weak operator topology. The proof of the next lemma is based on some of the estimates given by Axler and Zheng in [2].

Lemma 2.1 Let $S \in \mathfrak{L}\left(L_{a}^{2}\right)$. The following conditions are equivalent 
(a) $\left\|T_{B_{k}(S)}\right\| \leq C$, where $C>0$ does not depend on $k$.

(b) $\sup _{z \in D}\left|\left\langle\left(S_{z}-\left(T_{B_{k}(S)}\right)_{z}\right) f, g\right\rangle\right| \rightarrow 0$ for all $f, g \in L_{a}^{2}$.

(c) $T_{B_{k}(S)} \stackrel{\text { WOT }}{\rightarrow} S$.

Proof. If (b) holds then

$$
\left\langle\left(S-T_{B_{k}(S)}\right) U_{z} f, U_{z} g\right\rangle \rightarrow 0 \quad \text { for all } \quad f, g \in L_{a}^{2} \quad \text { and } \quad z \in D
$$

Since $U_{z}$ is a unitary operator, (c) holds. If (c) holds, a standard application of the BanachSteinhaus Theorem gives (a).

Now suppose that (a) holds. Writing $R_{k}=S-T_{B_{k}(S)}$, the hypothesis says that $\left\|R_{k}\right\| \leq C^{\prime}$ for some $C^{\prime}>0$ independent of $k$. Since

$$
K_{z}^{(0)}(w)=\sum_{m=0}^{\infty}(m+1) \bar{z}^{m} \omega^{m}
$$

for $z, \lambda \in D$ and $R \in \mathfrak{L}\left(L_{a}^{2}\right)$ we have

$$
B_{0}(R)\left(\varphi_{z}(\lambda)\right)=B_{0}\left(R_{z}\right)(\lambda)=\left(1-|\lambda|^{2}\right)^{2} \sum_{j, m=0}^{\infty}(j+1)(m+1)\left\langle R_{z} \omega^{j}, \omega^{m}\right\rangle \bar{\lambda}^{j} \lambda^{m}
$$

where the first equality holds by (1.4). Fix two nonnegative integers $n, j_{0}$. Then, for $0<\delta<1 / 2$ (to be chosen later) we obtain

$$
\begin{aligned}
\int_{\delta D} \frac{B_{0}(R)\left(\varphi_{z}(\lambda)\right) \bar{\lambda}^{n}}{\left(1-|\lambda|^{2}\right)^{2}} d A(\lambda) & =\sum_{j, m=0}^{\infty}(j+1)(m+1)\left\langle R_{z} \omega^{j}, \omega^{m}\right\rangle \int_{\delta D} \bar{\lambda}^{j+n} \lambda^{m} d A(\lambda) \\
& =\sum_{j=0}^{\infty}(j+1)\left\langle R_{z} \omega^{j}, \omega^{j+n}\right\rangle \delta^{2 j+2 n+2} \\
& =\delta^{2 n+2}\left(\sum_{j=0}^{j_{0}}(j+1)\left\langle R_{z} \omega^{j}, \omega^{j+n}\right\rangle \delta^{2 j}\right. \\
& \left.+\sum_{j=j_{0}+1}^{\infty}(j+1)\left\langle R_{z} \omega^{j}, \omega^{j+n}\right\rangle \delta^{2 j}\right) .
\end{aligned}
$$


Thus

$$
\begin{gathered}
\delta^{-2\left(n+j_{0}\right)-2} \int_{\delta D} \frac{B_{0}(R)\left(\varphi_{z}(\lambda)\right) \bar{\lambda}^{n}}{\left(1-|\lambda|^{2}\right)^{2}} d A(\lambda)-\sum_{j=0}^{j_{0}}(j+1)\left\langle R_{z} \omega^{j}, \omega^{j+n}\right\rangle \delta^{2\left(j-j_{0}\right)} \\
=\sum_{j=j_{0}+1}^{\infty}(j+1)\left\langle R_{z} \omega^{j}, \omega^{j+n}\right\rangle \delta^{2\left(j-j_{0}\right)} .
\end{gathered}
$$

Since $0<\delta<1 / 2$ and $\left\|\omega^{j}\right\|=(j+1)^{-1 / 2}$,

$$
\begin{aligned}
\left|\sum_{j=j_{0}+1}^{\infty}(j+1)\left\langle R_{z} \omega^{j}, \omega^{j+n}\right\rangle \delta^{2\left(j-j_{0}\right)}\right| & \leq\|R\| \sum_{j=j_{0}+1}^{\infty}(j+1)\left\|\omega^{j}\right\|\left\|\omega^{j+n}\right\| \delta^{2\left(j-j_{0}\right)} \\
& \leq \delta\|R\|,
\end{aligned}
$$

where the last inequality holds because $\sum_{j=1}^{\infty} \delta^{2 j} \leq \delta$ when $0<\delta<1 / 2$. Hence,

$$
\left|\delta^{-2\left(n+j_{0}\right)-2} \int_{\delta D} \frac{B_{0}(R)\left(\varphi_{z}(\lambda)\right) \bar{\lambda}^{n}}{\left(1-|\lambda|^{2}\right)^{2}} d A(\lambda)-\sum_{j=0}^{j_{0}}(j+1)\left\langle R_{z} \omega^{j}, \omega^{j+n}\right\rangle \delta^{2\left(j-j_{0}\right)}\right| \leq \delta\|R\|
$$

for all $z \in D, 0<\delta<1 / 2$, and nonnegative integers $n$ and $j_{0}$. Taking $R=R_{k}$ we have that the integral in the above expression tends to 0 uniformly in $z \in D$ whatever the choice of (fixed) $\delta, n$ and $j_{0}$. That is,

$$
\limsup _{k \rightarrow \infty} \sup _{z \in D}\left|\sum_{j=0}^{j_{0}}(j+1)\left\langle\left(R_{k}\right)_{z} \omega^{j}, \omega^{j+n}\right\rangle \delta^{2\left(j-j_{0}\right)}\right| \leq \delta C^{\prime}
$$

for every fixed $0<\delta<1 / 2$ and nonnegative integers $n$ and $j_{0}$. Putting $j_{0}=0$ we obtain that $\sup _{z \in D}\left|\left\langle\left(R_{k}\right)_{z} \omega^{0}, \omega^{0+n}\right\rangle\right| \rightarrow 0$. We can prove recursively that $\sup _{z \in D}\left|\left\langle\left(R_{k}\right)_{z} \omega^{j}, \omega^{j+n}\right\rangle\right| \rightarrow 0$ for every $0 \leq j \leq j_{0}$ and $n \geq 0$. Since $j_{0}$ and $n$ are arbitrary, we have

$$
\sup _{z \in D}\left|\left\langle\left(R_{k}\right)_{z} \omega^{j}, \omega^{m}\right\rangle\right| \rightarrow 0
$$

for every $j, m$, with $0 \leq j \leq m$. If we change $\bar{\lambda}^{n}$ by $\lambda^{n}$ in the integrand of (2.1), we obtain that the above holds for every $j, m$, with $0 \leq m \leq j$. Thus, $\sup _{z \in D}\left|\left\langle\left(R_{k}\right)_{z} p, q\right\rangle\right| \rightarrow 0$ for every polynomials $p$ and $q$. Since the polynomials are dense in $L_{a}^{2}$ and $\left\|\left(R_{k}\right)_{z}\right\|=\left\|R_{k}\right\| \leq C^{\prime}$ for all $z \in D$, (b) follows.

The next result is in [10], Lemma 5.6. 
Lemma 2.2 Let $\left\{S_{k}\right\}$ be a sequence in $\mathfrak{L}\left(L_{a}^{2}\right)$ such that for some $p>4$,

(1) $\left\|B_{0}\left(S_{k}\right)\right\|_{\infty} \rightarrow 0$ when $k \rightarrow \infty$,

(2) $\sup _{z \in D}\left\|\left(S_{k}\right)_{z} 1\right\|_{p} \leq C$ and $\sup _{z \in D}\left\|\left(S_{k}^{*}\right)_{z} 1\right\|_{p} \leq C$,

where $C>0$ does not depend on $k$. Then $\left\|S_{k}\right\|_{\mathfrak{L}\left(L_{a}^{2}\right)} \rightarrow 0$ when $k \rightarrow \infty$.

Corollary 2.3 Let $S \in \mathfrak{L}\left(L_{a}^{2}\right)$ such that for some $p>4$,

$$
\sup _{z \in D}\left\|S_{z} 1-\left(T_{B_{k}(S)}\right)_{z} 1\right\|_{p} \leq C \text { and } \sup _{z \in D}\left\|S_{z}^{*} 1-\left(T_{B_{k}\left(S^{*}\right)}\right)_{z} 1\right\|_{p} \leq C,
$$

where $C>0$ is independent of $k$. Then $T_{B_{k}(S)} \rightarrow S$ in $\mathfrak{L}\left(L_{a}^{2}\right)$-norm.

Proof. Write $S_{k}=S-T_{B_{k}(S)}$. Since (1.3) says that $B_{0} B_{k}=B_{k} B_{0}$ on $\mathfrak{L}\left(L_{a}^{2}\right)$,

$$
B_{0}\left(S_{k}\right)=B_{0}(S)-B_{0}\left(T_{B_{k}(S)}\right)=B_{0}(S)-B_{0}\left(B_{k}(S)\right)=B_{0}(S)-B_{k}\left(B_{0}(S)\right),
$$

which tends uniformly to 0 when $k \rightarrow \infty$ because $B_{0}(S) \in \mathcal{A}$. That is, $\left\{S_{k}\right\}$ satisfies (1) of Lemma 2.2. Since (2) of Lemma 2.2 is our hypothesis, the lemma applies.

Theorem 2.4 Let $S \in \mathfrak{L}\left(L_{a}^{2}\right)$. If there is $p>4$ such that

$$
\sup _{z \in D}\left\|T_{B_{k}(S) \circ \varphi_{z}} 1\right\|_{p}<C \text { and } \sup _{z \in D}\left\|T_{B_{k}(S) \circ \varphi_{z}}^{*} 1\right\|_{p}<C,
$$

where $C>0$ is independent of $k$, then $T_{B_{k}(S)} \rightarrow S$ in $\mathfrak{L}\left(L_{a}^{2}\right)$-norm.

Proof. By Corollary 2.3 we only need to show that the theorem's hypotheses imply (2.2). Since $T_{B_{k}(S) \circ \varphi_{z}}=\left(T_{B_{k}(S)}\right)_{z}$ and

$$
T_{B_{k}(S) \circ \varphi_{z}}^{*}=T_{\overline{B_{k}\left(S_{z}\right)}}=T_{B_{k}\left(S_{z}^{*}\right)}=T_{B_{k}\left(S^{*}\right) \varphi_{z}}=\left(T_{B_{k}\left(S^{*}\right)}\right)_{z},
$$

we only have to prove the first of the two inequalities

$$
\sup _{z \in D}\left\|S_{z} 1\right\|_{p}<\infty \text { and } \sup _{z \in D}\left\|S_{z}^{*} 1\right\|_{p}<\infty
$$

because the second one will follow by symmetry.

By Lemma 5.3 of [10],

$$
\left\|T_{B_{k}(S)}\right\|_{\mathfrak{L}\left(L_{a}^{2}\right)} \leq C_{p} \sup _{z \in D}\left\|T_{B_{k}(S) \circ \varphi_{z}} 1\right\|_{p}^{1 / 2} \sup _{\omega \in D}\left\|T_{B_{k}(S) \circ \varphi_{\omega}}^{*} 1\right\|_{p}^{1 / 2},
$$


where $C_{p}$ depends only on $p$. By hypothesis then $\left\|T_{B_{k}(S)}\right\|_{\mathfrak{L}\left(L_{a}^{2}\right)}$ is bounded independently of $k$, and the equivalence between (a) and (b) of Lemma 2.1 says that

$$
\sup _{z \in D}\left|\left\langle S_{z}-\left(T_{B_{k}(S)}\right)_{z} f, g\right\rangle\right| \rightarrow 0 \text { for all } f, g \in L_{a}^{2}
$$

Taking $f=1$ and $g$ a polynomial with $\|g\|_{q}=1$ (where $1 / p+1 / q=1$ ), we see that for any $\varepsilon>0$ and $z_{0} \in D$ :

$$
\begin{aligned}
\left|\left\langle S_{z_{0}} 1, g\right\rangle\right| & \leq \sup _{z \in D}\left|\left\langle S_{z}-\left(T_{B_{k}(S)}\right)_{z} 1, g\right\rangle\right|+\left|\left\langle\left(T_{B_{k}(S)}\right)_{z_{0}} 1, g\right\rangle\right| \\
& \leq \varepsilon+\left\|\left(T_{B_{k}(S)}\right)_{z_{0}} 1\right\|_{p} \leq \varepsilon+C
\end{aligned}
$$

if $k$ is big enough, where $C>0$ is given by hypothesis and does not depend on $k$. Since $\varepsilon$ is arbitrary, we obtain that $\left\|S_{z_{0}} 1\right\|_{p} \leq C$ for every $z_{0} \in D$, which proves the theorem.

All the measures considered in the paper will be Borel regular measures. If $\mu$ is a finite measure on $D$ and $z \in D$, write

$$
B_{0}(\mu)(z)=\int_{D} \frac{\left(1-|z|^{2}\right)^{2}}{|1-z \bar{w}|^{4}} d \mu(w)
$$

The formula

$$
T_{\mu} f(z)=\int_{D} \frac{f(w)}{(1-\bar{w} z)^{2}} d \mu(w)
$$

defines an analytic function for every polynomial $f$. When $\mu \geq 0$ and $1<p<\infty$, a necessary and sufficient condition for $T_{\mu}$ to be continuous from $L_{a}^{p}$ into $L_{a}^{p}$ is that $\left\|B_{0}(\mu)\right\|_{\infty}<\infty$. Such measure $\mu$ is called a Carleson measure (on Bergman spaces), and it is known that $\left\|T_{\mu}\right\|_{\mathfrak{L}\left(L_{a}^{p}\right)} \leq A_{p}\left\|B_{0}(\mu)\right\|_{\infty}$, where $A_{p}>0$ only depends on $p$ (see the proofs in [12], Ch. 6).

If $\mu$ is a complex measure, it can be decomposed in a standard way as $\mu=\mu_{1}-\mu_{2}+$ $i\left(\mu_{3}-\mu_{4}\right)$, where $\mu_{j} \geq 0$ for $j=1, \ldots, 4$, and $|\mu|=\mu_{1}+\mu_{2}+\mu_{3}+\mu_{4}$. Thus, if $|\mu|$ is a Carleson measure then $T_{\mu} \in \mathfrak{L}\left(L_{a}^{p}\right)$ and

$$
\left\|T_{\mu}\right\|_{\mathfrak{L}\left(L_{a}^{p}\right)} \leq \sum_{j=1}^{4}\left\|T_{\mu_{j}}\right\|_{\mathfrak{L}\left(L_{a}^{p}\right)} \leq A_{p} \sum_{j=1}^{4}\left\|B_{0}\left(\mu_{j}\right)\right\|_{\infty} \leq 4 A_{p}\left\|B_{0}(|\mu|)\right\|_{\infty}
$$

A simple application of Fubini's theorem gives

$$
\left\langle T_{\mu} f, g\right\rangle=\int_{D} f(w) \overline{g(w)} d \mu(w)
$$


for every $f, g \in H^{\infty}$. An immediate consequence is that $B_{0}(\mu)=B_{0}\left(T_{\mu}\right)$.

In Theorem 5.7 of [10] we showed that $T_{B_{n}(a)} \rightarrow T_{a}$ in $\mathfrak{L}\left(L_{a}^{2}\right)$-norm for any $a \in L^{\infty}$. The next result generalizes that theorem.

Corollary 2.5 Let $\mu$ be a measure on $D$ such that $|\mu|$ is Carleson on Bergman spaces. Then $T_{B_{k}\left(T_{\mu}\right)} \rightarrow T_{\mu}$ in $\mathfrak{L}\left(L_{a}^{2}\right)$-norm.

Proof. By the comments preceding the corollary, it is enough to assume that $\mu \geq 0$. Since

$$
\begin{aligned}
\left(B_{n} T_{\mu}\right)(z) & =(n+1)\left(1-|z|^{2}\right)^{2+n} \sum_{j=0}^{n}\left(\begin{array}{l}
n \\
j
\end{array}\right)(-1)^{j}\left\langle T_{\mu}\left(\omega^{j} K_{z}^{(n)}\right), \omega^{j} K_{z}^{(n)}\right\rangle \\
& =(n+1)\left(1-|z|^{2}\right)^{2+n} \sum_{j=0}^{n}\left(\begin{array}{l}
n \\
j
\end{array}\right)(-1)^{j} \int_{D} \frac{|\omega|^{2 j}}{|1-\bar{z} \omega|^{2(2+n)}} d \mu(\omega) \\
& =\int_{D} \frac{\left(1-|z|^{2}\right)^{2+n}}{|1-\bar{z} \omega|^{2(2+n)}}(n+1)\left(1-|\omega|^{2}\right)^{n} d \mu(\omega),
\end{aligned}
$$

$B_{n}\left(T_{\mu}\right) \geq 0$, and since

$$
B_{0}\left(B_{n}\left(T_{\mu}\right) \circ \varphi_{z}\right)=B_{0} B_{n}\left(\left(T_{\mu}\right)_{z}\right)=B_{n} B_{0}\left(\left(T_{\mu}\right)_{z}\right)=B_{n}\left(B_{0}\left(T_{\mu}\right) \circ \varphi_{z}\right),
$$

we have

$$
\left\|B_{0}\left(B_{n}\left(T_{\mu}\right) \circ \varphi_{z}\right)\right\|_{\infty}=\left\|B_{n}\left(B_{0}\left(T_{\mu}\right) \circ \varphi_{z}\right)\right\|_{\infty} \leq\left\|B_{0}\left(T_{\mu}\right) \circ \varphi_{z}\right\|_{\infty}=\left\|B_{0}\left(T_{\mu}\right)\right\|_{\infty} .
$$

Hence, $\left(B_{n}\left(T_{\mu}\right) \circ \varphi_{z}\right) d A$ is a Carleson measure, and

$$
\left\|T_{B_{n}\left(T_{\mu}\right) \circ \varphi_{z}}\right\|_{\mathfrak{L}\left(L_{a}^{p}\right)} \leq A_{p}\left\|B_{0}\left(T_{\mu}\right)\right\|_{\infty}
$$

for every integer $n \geq 0, z \in D$ and $1<p<\infty$. Since $T_{B_{n}\left(T_{\mu}\right) \circ \varphi_{z}}$ is self-adjoint, Theorem 2.4 implies that $T_{B_{n}\left(T_{\mu}\right)} \rightarrow T_{\mu}$.

The next lemma is well-known. Since I did not find it explicitly stated in the literature, a proof is sketched here.

Lemma 2.6 Let $\mu$ be a finite positive measure on $\bar{D}$. If

$$
\sup _{z \in D} \int_{\bar{D}} \frac{\left(1-|z|^{2}\right)^{2}}{|1-z \bar{w}|^{4}} d \mu(w)<\infty
$$

then $\mu(\partial D)=0$. 
Proof. For integers $N \geq 2$ and $0 \leq j \leq N-1$, write

$$
I_{j}=\left\{e^{i \theta}: \frac{2 \pi j}{N} \leq \theta<\frac{2 \pi(j+1)}{N}\right\} \quad \text { and } \quad z_{j}=\left(1-\frac{1}{N}\right) e^{i \pi\left(\frac{2 j+1}{N}\right)} .
$$

That is, $z_{j} /\left|z_{j}\right|$ is the middle point of $I_{j}$ and $2 \pi\left(1-\left|z_{j}\right|\right)$ is the length of $I_{j}$. It is geometrically clear that there is some absolute constant $c>0$ such that

$$
w \in I_{j} \Rightarrow\left|w-z_{j}\right| \leq c\left(1-\left|z_{j}\right|\right)
$$

So, if $s$ denotes the supremun in (2.4),

$$
s \geq \int_{I_{j}} \frac{\left(1-\left|z_{j}\right|\right)^{2}}{\left|1-z_{j} \bar{w}\right|^{4}} d \mu(w)=\int_{I_{j}} \frac{\left(1-\left|z_{j}\right|\right)^{2}}{\left|w-z_{j}\right|^{4}} d \mu(w) \geq \frac{1}{c^{4}} \frac{1}{\left(1-\left|z_{j}\right|\right)^{2}} \mu\left(I_{j}\right)
$$

for all $j$. Consequently,

$$
\mu(\partial D)=\sum_{j=0}^{N-1} \mu\left(I_{j}\right) \leq s c^{4} \sum_{j=0}^{N-1}\left(1-\left|z_{j}\right|\right)^{2}=s c^{4} \frac{1}{N} \rightarrow 0
$$

as $N \rightarrow \infty$.

Theorem 2.7 Let $S \in \mathfrak{L}\left(L_{a}^{2}\right)$. Then

(1) $S=T_{\mu}$ for a Carleson measure $\mu \geq 0$ if and only if $B_{n}(S) \geq 0$ for all $n$.

(2) $S=T_{a}$ for $a \in L^{\infty}$ if and only if $\left\|B_{n}(S)\right\|_{\infty} \leq C$, with $C$ independent of $n$.

Proof. If $\mu$ is a positive Carleson measure, $B_{n}\left(T_{\mu}\right) \geq 0$ by (2.3). Suppose now that $B_{n}(S) \geq 0$ for every $n$, and consider the measures $\mu_{n}=B_{n}(S) d A$. Let $\mathcal{M}(\bar{D})$ denote the space of finite measure on $\bar{D}$ with the norm $\|\nu\|=|\nu(\bar{D})|$. That is, $\mathcal{M}(\bar{D})$ is the dual space of $C(\bar{D})$, the space of continuous functions on $\bar{D}$. By (1.3),

$$
\left\|B_{0}\left(\mu_{n}\right)\right\|_{\infty}=\left\|B_{0} B_{n}(S)\right\|_{\infty}=\left\|B_{n} B_{0}(S)\right\|_{\infty} \leq\left\|B_{0}(S)\right\|_{\infty} \leq\|S\| .
$$

Hence, $\mu_{n}$ is a Carleson measure with $\mathcal{M}(\bar{D})$-norm $\left\|\mu_{n}\right\|=\mu_{n}(D)=B_{0}\left(\mu_{n}\right)(0) \leq\|S\|$ for all $n$. By the Banach-Alaoglu Theorem (see for instance [11, p. 29]) there is a subsequence $\mu_{n_{k}}$ and $\mu \in \mathcal{M}(\bar{D})$ such that $\mu_{n_{k}} \rightarrow \mu$ in the weak-star topology of $\mathcal{M}(\bar{D})$. This means that

$$
\int_{\bar{D}} f d \mu_{n_{k}} \rightarrow \int_{\bar{D}} f d \mu, \quad \forall f \in C(\bar{D}) .
$$


It is clear that $\mu \geq 0$ and $\|\mu\| \leq\|S\|$. If $z \in D$ is fixed and we take $f(w)=\left(1-|z|^{2}\right)^{2} /|1-\bar{z} w|^{4}$ in (2.6), we get

$$
B_{0}\left(\mu_{n_{k}}\right)=\int_{\bar{D}} \frac{\left(1-|z|^{2}\right)^{2}}{|1-z \bar{w}|^{4}} d \mu_{n_{k}}(w) \rightarrow \int_{\bar{D}} \frac{\left(1-|z|^{2}\right)^{2}}{|1-z \bar{w}|^{4}} d \mu(w)
$$

which together with (2.5) implies that the last integral is bounded by $\|S\|$. Now Lemma 2.6 tells us that $\mu(\partial D)=0$, and consequently the last integral is $B_{0}(\mu)(z)$, which is bounded by $\|S\|$. In particular, we have that $T_{\mu}$ defines a bounded operator on $L_{a}^{2}$.

If $p, q$ are two polynomials and we take $f=p \bar{q}$ in (2.6), we see that $\left\langle T_{\mu_{n_{k}}} p, q\right\rangle \rightarrow\left\langle T_{\mu} p, q\right\rangle$. Since the polynomials are dense in $L_{a}^{2}$ and by $(2.5),\left\|T_{\mu_{n}}\right\| \leq A_{2}\left\|B_{0}\left(\mu_{n}\right)\right\|_{\infty} \leq A_{2}\|S\|$, we deduce that $T_{\mu_{n_{k}}} \stackrel{\text { WOT }}{\rightarrow} T_{\mu}$. But the above inequalities and Lemma 2.1 also imply that $T_{\mu_{n}} \stackrel{\text { WOT }}{\rightarrow} S$, so $S=T_{\mu}$. This proves $(1)$.

The proof of (2) follows the same lines, but it is simpler. If $S=T_{a}$ then $\left\|B_{n}\left(T_{a}\right)\right\|_{\infty}=$ $\left\|B_{n}(a)\right\|_{\infty} \leq\|a\|_{\infty}$. If $\left\|B_{n}(S)\right\|_{\infty} \leq C$ for all $n$, there is a subsequence $\left\{n_{k}\right\}$ such that $B_{n_{k}}(S)$ converges in the weak-star topology of $L^{\infty}$ to some function $a$, with $\|a\|_{\infty} \leq C$. In particular, for every $f, g \in L_{a}^{2}$,

$$
\left\langle T_{B_{n_{k}}(S)} f, g\right\rangle=\int B_{n_{k}}(S) f \bar{g} d A \rightarrow \int a f \bar{g} d A=\left\langle T_{a} f, g\right\rangle .
$$

Hence, $T_{B_{n_{k}}(S)} \stackrel{\text { WOT }}{\longrightarrow} T_{a}$, but since $\left\|T_{B_{n}(S)}\right\| \leq\left\|B_{n}(S)\right\|_{\infty} \leq C$, Lemma 2.1 says that $T_{B_{n}(S)} \stackrel{\text { WOT }}{\longrightarrow} S$.

Remark 2.8 It is clear from the proof of Theorem 2.7 that in (1) or (2) the quantifier 'for all $n$ ' can be replaced by 'for infinitely many values of $n$ '. In particular, taking into account Corollary 2.5, we see that if $S \notin \mathfrak{T}\left(L^{\infty}\right)$ then there are at most finitely many $n$ 's such that $B_{n}(S) \geq 0$ or $\left\|B_{n}(S)\right\|_{\infty} \leq C$, for any given $C>0$.

\section{Radial operators}

We will say that $S \in \mathfrak{L}\left(L_{a}^{2}\right)$ is a radial operator if it is diagonal with respect to the orthonormal base $\left\{\sqrt{n+1} z^{n}: n \geq 0\right\}$. That is, $S z^{n}=\lambda_{n}(S) z^{n}$, where $\left\{\lambda_{n}(S)\right\}$ is a bounded sequence. Clearly, every bounded sequence defines a radial operator. The name radial originates in the fact that if $a \in L^{\infty}$ is a radial function (i.e.: $\left.a(z)=a(|z|)\right)$ then $T_{a}$ is a radial operator. The set of radial operators form a commutative $C^{*}$-subalgebra of $\mathfrak{L}\left(L_{a}^{2}\right)$. 
We begin by showing the elementary fact that if $S$ is radial, then so is $B_{n}(S)$. Using that when $S$ is a radial operator,

$$
\left\langle S w^{j}, w^{k}\right\rangle= \begin{cases}0 & \text { if } j \neq k \\ \lambda_{j}(S) /(j+1) & \text { if } j=k\end{cases}
$$

and

$$
\frac{1}{(1-w \bar{z})^{2+n}}=\sum_{m=0}^{\infty}\left(\begin{array}{c}
m+n+1 \\
m
\end{array}\right)(\bar{z} w)^{m}
$$

we obtain

$$
\begin{aligned}
\left\langle S\left(w^{j} K_{z}^{(n)}\right), w^{j} K_{z}^{(n)}\right\rangle & =\sum_{m_{1}, m_{2}=0}^{\infty}\left(\begin{array}{c}
m_{1}+n+1 \\
m
\end{array}\right)\left(\begin{array}{c}
m_{2}+n+1 \\
m
\end{array}\right) \bar{z}^{m_{1}} z^{m_{2}}\left\langle S w^{j+m_{1}}, w^{j+m_{2}}\right\rangle \\
& =\sum_{m=0}^{\infty}\left(\begin{array}{c}
m+n+1 \\
m
\end{array}\right)^{2}|z|^{2 m} \frac{\lambda_{j+m}(S)}{(j+m+1)}
\end{aligned}
$$

Therefore

$$
B_{n}(S)(z)=(n+1)\left(1-|z|^{2}\right)^{2+n} \sum_{j=0}^{n} \sum_{m=0}^{\infty}\left(\begin{array}{c}
n \\
j
\end{array}\right)(-1)^{j}\left(\begin{array}{c}
m+n+1 \\
m
\end{array}\right)^{2}|z|^{2 m} \frac{\lambda_{j+m}(S)}{(j+m+1)}
$$

For $t$ a real number, let $C_{t}$ be the composition operator $C_{t} f(z)=f\left(e^{i t} z\right)$. Clearly $C_{t}$ is a unitary operator with $C_{t}^{*}=C_{-t}$. If $S \in \mathfrak{L}\left(L_{a}^{2}\right)$, the 'radialization' of $S$ is

$$
\tilde{S} \stackrel{\text { def }}{=} \int_{0}^{2 \pi} C_{-t} S C_{t} \frac{d t}{2 \pi}
$$

where the integral is taken in the weak sense. Then

$$
\left\langle\tilde{S} w^{j}, w^{k}\right\rangle=\int_{0}^{2 \pi} e^{i(j-k) t}\left\langle S w^{j}, w^{k}\right\rangle \frac{d t}{2 \pi}= \begin{cases}0 & \text { if } j \neq k \\ \left\langle S w^{j}, w^{j}\right\rangle & \text { if } j=k\end{cases}
$$

Hence, $\tilde{S}$ is a radial operator and $\tilde{S}=S$ when $S$ is radial. The above equality implies that every bounded operator can be written in a unique way as $S=S_{1}+S_{2}$, where $S_{1}$ is radial, $\left\langle S_{1} w^{j}, w^{j}\right\rangle=\left\langle S w^{j}, w^{j}\right\rangle$, and $S_{2} w^{j}$ is orthogonal to $w^{j}$ for every $j$. Clearly, the decomposition is $S_{1}=\tilde{S}$ and $S_{2}=S-\tilde{S}$.

If $a \in L^{\infty}$ and $f, g \in L_{a}^{2}$ then

$$
\left\langle C_{-t} T_{a} C_{t} f, g\right\rangle=\int_{D} a(w) f\left(e^{i t} w\right) \overline{g\left(e^{i t} w\right)} d A(w)=\int_{D} a\left(e^{i t} w\right) f(w) \overline{g(w)} d A(w) .
$$


Thus, $C_{-t} T_{a} C_{t}=T_{a \circ r_{t}}$, where $r_{t}(z)=e^{i t} z$, and consequently

$$
C_{-t} T_{a_{1}} \ldots T_{a_{m}} C_{t}=\left(C_{-t} T_{a_{1}} C_{t}\right) C_{-t} \ldots C_{t}\left(C_{-t} T_{a_{m}} C_{t}\right)=T_{a_{1} \circ r_{t}} \ldots T_{a_{m} \circ r_{t}}
$$

for $a_{1}, \ldots, a_{m} \in L^{\infty}$. Also, $\widetilde{T}_{a}=T_{\tilde{a}}$, where $\tilde{a}$ denotes the radialization of the function $a$ :

$$
\tilde{a}(z) \stackrel{\text { def }}{=} \int_{0}^{2 \pi} a\left(e^{i t} z\right) \frac{d t}{2 \pi} .
$$

The next lemma provides a very useful formula for $T_{B_{n}(S)}$ when $S$ is a radial operator. We recall that $d A_{n}(w)=(n+1)\left(1-|w|^{2}\right)^{n} d A(w)$.

Lemma 3.1 Let $S \in \mathfrak{L}\left(L_{a}^{2}\right)$ be a radial operator. Then $T_{B_{n}(S)}=\int_{D} S_{w} d A_{n}(w)$.

Proof. First we prove the result for $S=T_{a}$, where $a \in L^{\infty}$ is a radial function. If $f, g \in L_{a}^{2}$ then

$$
\begin{aligned}
\left\langle B_{n}\left(T_{a}\right) f, g\right\rangle & =\iint a\left(\varphi_{z}(w)\right) f(z) \overline{g(z)} d A_{n}(w) d A(z) \\
& =\iint a\left(\varphi_{w}(z)\right) f(z) \overline{g(z)} d A_{n}(w) d A(z) \\
& =\int\left\langle\left(T_{a}\right)_{w} f, g\right\rangle_{d A} d A_{n}(w) \\
& \left.=\left\langle\iint\left(T_{a}\right)_{w} d A_{n}(w)\right] f, g\right\rangle_{d A},
\end{aligned}
$$

where the second equality holds because $\left|\varphi_{w}(z)\right|=\left|\varphi_{z}(w)\right|$ and $a$ is radial.

Now let $S$ be a general radial operator. A result of Englis (see [5] or [6]) states that $\left\{T_{a}: a \in L^{\infty}\right\}$ is dense in $\mathfrak{L}\left(L_{a}^{2}\right)$ with the strong operator topology. Hence, there are $a_{k} \in L^{\infty}$ such that $T_{a_{k}} \stackrel{\text { WOT }}{\rightarrow} S$. By the Banach-Steinhaus Theorem, $\left\|T_{a_{k}}\right\| \leq C$ independently of $k$. So, taking radializations and using that $\widetilde{T}_{a_{k}}=T_{\tilde{a}_{k}}$, the dominated convergence theorem gives

$$
\left\langle T_{\tilde{a}_{k}} f, g\right\rangle=\int_{0}^{2 \pi}\left\langle T_{a_{k}} C_{t} f, C_{t} g\right\rangle \frac{d t}{2 \pi} \rightarrow \int_{0}^{2 \pi}\left\langle S C_{t} f, C_{t} g\right\rangle \frac{d t}{2 \pi}=\langle\tilde{S} f, g\rangle
$$

when $k \rightarrow \infty$ for every $f, g \in L_{a}^{2}$. That is, we can assume that the functions $a_{k}$ are radial. Let $n$ be an arbitrary fixed nonnegative integer. It follows from the definition of $B_{n}$ that $B_{n}\left(T_{a_{k}}\right) \rightarrow B_{n}(S)$ pointwise on $D$ when $k \rightarrow \infty$. Since (1.1) says that

$$
\left\|B_{n}\left(T_{a_{k}}\right)\right\|_{\infty} \leq(n+1) 2^{n}\left\|T_{a_{k}}\right\| \leq(n+1) 2^{n} C
$$


two new applications of the dominated convergence theorem yield

$$
\begin{aligned}
\left\langle B_{n}(S) f, g\right\rangle & =\lim _{k}\left\langle B_{n}\left(T_{a_{k}}\right) f, g\right\rangle \\
& =\lim _{k}\left\langle\left[\int\left(T_{a_{k}}\right)_{w} d A_{n}(w)\right] f, g\right\rangle \\
& =\lim _{k} \int\left\langle\left(T_{a_{k}}\right)_{w} f, g\right\rangle d A_{n}(w) \\
& =\int\left\langle S_{w} f, g\right\rangle d A_{n}(w) \\
& =\left\langle\left[\int S_{w} d A_{n}(w)\right] f, g\right\rangle
\end{aligned}
$$

where the second equality holds because the lemma was already proved for Toeplitz operators.

Corollary 3.2 Let $S \in \mathfrak{L}\left(L_{a}^{2}\right)$ be a radial operator and $n$ be a nonnegative integer. Then

(1) $\left\|T_{B_{n}(S)}\right\| \leq\|S\|$,

(2) $T_{B_{n}(S)} \geq 0$ if $S \geq 0$.

(3) $T_{B_{n}(S)} \stackrel{\text { WOT }}{\rightarrow} S$ when $n \rightarrow \infty$.

Proof. By Lemma 3.1,

$$
\left\|T_{B_{n}(S)}\right\|=\left\|\int_{D} S_{w} d A_{n}(w)\right\| \leq \int\left\|S_{w}\right\| d A_{n}(w)=\|S\|,
$$

where the last equality holds because, since $U_{w}$ is unitary and self-adjoint, $\left\|U_{w} S U_{w}\right\|=\|S\|$, and $d A_{n}$ is a probability measure. Since $S_{w} \geq 0$ when $S \geq 0,(2)$ follows by a similar use of the formula in Lemma 3.1. Finally (3) is a consequence of (1) and Lemma 2.1.

We are now ready to prove the second main result of this paper.

Theorem 3.3 Let $S \in \mathfrak{L}\left(L_{a}^{2}\right)$ be a radial operator. The following conditions are equivalent.

(1) $S \in \mathfrak{T}\left(L^{\infty}\right)$,

(2) $T_{B_{n}(S)} \rightarrow S$ in operator norm, 
(3) $F:(D,||) \rightarrow\left(\mathfrak{L}\left(L_{a}^{2}\right),\|\|\right)$ given by $F(w)=S_{w}$ is continuous,

(4) $F$ is continuous in 0 .

Proof. $(1) \Rightarrow(2)$. Since $S \in \mathfrak{T}\left(L^{\infty}\right)$ there is a sequence of operators $S_{k} \rightarrow S$, where each $S_{k}$ is a finite sum of finite products of Toeplitz operators with bounded symbols. Since the process of radialization is continuous and $S$ is radial, $\tilde{S}_{k} \rightarrow \tilde{S}=S$. Corollary 3.2 now tells us that for every fixed nonnegative integer $n$,

$$
\left\|T_{B_{n}\left(\tilde{S}_{k}\right)}-T_{B_{n}(S)}\right\|=\left\|T_{B_{n}\left(\tilde{S}_{k}-S\right)}\right\| \leq\left\|\tilde{S}_{k}-S\right\| \rightarrow 0
$$

when $k \rightarrow \infty$. Moreover, since

$$
\begin{aligned}
\left\|S-T_{B_{n}(S)}\right\| & \leq\left\|S-\tilde{S}_{k}\right\|+\left\|\tilde{S}_{k}-T_{B_{n}\left(\tilde{S}_{k}\right)}\right\|+\left\|T_{B_{n}\left(\tilde{S}_{k}\right)}-T_{B_{n}(S)}\right\| \\
& \leq 2\left\|S-\tilde{S}_{k}\right\|+\left\|\tilde{S}_{k}-T_{B_{n}\left(\tilde{S}_{k}\right)}\right\|,
\end{aligned}
$$

it is enough to prove (2) for $\tilde{S}_{k}$, but since $S_{k}$ is a finite sum of finite products of Toeplitz operators, the proof reduces to show that if

$$
Q=\int_{0}^{2 \pi} T_{a_{1} \circ r_{t}} \ldots T_{a_{m} \circ r_{t}} \frac{d t}{2 \pi} \text {, with } a_{1}, \ldots, a_{m} \in L^{\infty},
$$

then $T_{B_{n}(Q)} \rightarrow Q$. By Lemma 3.1,

$$
\begin{aligned}
T_{B_{n}(Q)} & =\int_{D} U_{w}\left(\int_{0}^{2 \pi} T_{a_{1} \circ r_{t}} \ldots T_{a_{m} \circ r_{t}} \frac{d t}{2 \pi}\right) U_{w} d A_{n}(w) \\
& =\int_{D} \int_{0}^{2 \pi} T_{a_{1} \circ r_{t} \circ \varphi_{w}} \ldots T_{a_{m} \circ r_{t} \circ \varphi_{w}} \frac{d t}{2 \pi} d A_{n}(w) .
\end{aligned}
$$

Consequently, for any $z \in D$,

$$
T_{B_{n}(Q) \circ \varphi_{z}}=U_{z} T_{B_{n}(Q)} U_{z}=\int_{D} \int_{0}^{2 \pi} T_{b_{1}} \ldots T_{b_{m}} \frac{d t}{2 \pi} d A_{n}(w)
$$

where $b_{j}=a_{j} \circ r_{t} \circ \varphi_{w} \circ \varphi_{z}$ for $j=1, \ldots, m$.

If $1<p<\infty$ and we look at each $T_{b_{j}}$ as an operator on $L_{a}^{p}$, we have

$$
\left\|T_{b_{j}}\right\|_{\mathfrak{L}\left(L_{a}^{p}\right)} \leq C_{p}\left\|b_{j}\right\|_{\infty}=C_{p}\left\|a_{j}\right\|_{\infty}
$$


for $1 \leq j \leq m$, where $C_{p}$ is the norm of the Bergman projection from $L^{p}(d A)$ into $L_{a}^{p}$. Since $(2 \pi)^{-1} d t d A_{n}(w)$ is a probability measure on $[0,2 \pi] \times D$, the above estimate and (3.1) yield

$$
\left\|T_{B_{n}(Q) \circ \varphi_{z}}\right\|_{\mathfrak{L}\left(L_{a}^{p}\right)} \leq C_{p}^{m}\left\|a_{1}\right\|_{\infty} \ldots\left\|a_{m}\right\|_{\infty},
$$

where the right member does not depend on $z$ or $n$. Since $T_{B_{n}(Q) \circ \varphi_{z}}^{*}$ satisfies an equality as (3.1) with each $b_{j}$ replaced by $\bar{b}_{j}$, the last estimate also holds for $T_{B_{n}(Q) \circ \varphi_{z}}^{*}$. Hence, Theorem 2.4 tells us that $T_{B_{n}(Q)} \rightarrow Q$ in $\mathfrak{L}\left(L_{a}^{2}\right)$-norm and completes the proof of (2).

$(2) \Rightarrow(3)$. By $(2)$ it is enough to prove that the map $w \mapsto\left(T_{B_{n}(S)}\right)_{w}$ is continuous for every $n$. Moreover, since (1.2) says that $B_{n}(S) \in \mathcal{A}$, we must prove that $w \mapsto T_{a \circ \varphi_{w}}$ is continuous when $a \in \mathcal{A}$ is radial. Let $\varepsilon>0$. Since $a \in \mathcal{A}$, there is some $\delta>0$ depending only on $\varepsilon$ such that

$$
\left|a\left(w_{1}\right)-a\left(w_{2}\right)\right|<\varepsilon \text { if } \rho\left(w_{1}, w_{2}\right)<\delta .
$$

For $w, w_{0} \in D$ we have

$$
\begin{aligned}
\left\|T_{a \circ \varphi_{w}}-T_{a \circ \varphi_{w_{0}}}\right\| & \leq \sup _{z \in D}\left|a\left(\varphi_{w}(z)\right)-a\left(\varphi_{w_{0}}(z)\right)\right| \\
& =\sup _{z \in D} \mid a\left(\varphi_{z}(w)\right)-a\left(\varphi_{z}\left(w_{0}\right) \mid<\varepsilon\right.
\end{aligned}
$$

if $\rho\left(w, w_{0}\right)<\delta$ by (3.2), because $\rho\left(\varphi_{z}(w), \varphi_{z}\left(w_{0}\right)\right)=\rho\left(w, w_{0}\right)$ for every $z \in D$. The easy inequality $\left(1-\left|w_{0}\right|\right) \rho\left(w, w_{0}\right) \leq\left|w-w_{0}\right|$ then gives (3).

Since (3) $\Rightarrow(4)$ and $(2) \Rightarrow(1)$ are trivial, only (4) $\Rightarrow(2)$ needs to be proved. So, suppose that (4) holds. First observe that since $S$ is radial and

$$
U_{0} f(z)=f\left(\varphi_{0}(z)\right) \varphi_{0}^{\prime}(z)=-f(-z)
$$

for $f \in L_{a}^{2}$, then $S_{0}=U_{0} S U_{0}=S$. By Lemma 3.1,

$$
T_{B_{n}(S)}-S=\int_{D}\left(S_{w}-S\right) d A_{n}(w)=\int_{\{|w|<\delta\}}\left(S_{w}-S\right) d A_{n}(w)+\int_{\{|w| \geq \delta\}}\left(S_{w}-S\right) d A_{n}(w)
$$

for $0<\delta<1$. The norm of the second integral in the sum is bounded by

$$
\int_{\{|w| \geq \delta\}}\left\|S_{w}-S\right\| d A_{n}(w) \leq 2\|S\| \int_{\{|w| \geq \delta\}} d A_{n}(w) \rightarrow 0
$$

as $n \rightarrow \infty$, because the mass of the measures tend to concentrate at 0 . The norm of the first integral in the sum is bounded by $\sup _{|w|<\delta}\left\|S_{w}-S\right\|$, which can be made arbitrarily small 
by taking $\delta$ small, since by hypothesis $(4), S_{w} \rightarrow S_{0}=S$ when $w \rightarrow 0$.

Let $R a d$ denote the algebra of bounded radial operators. An immediate consequence of Theorem 3.3 is that the space $\left\{T_{a}: a\right.$ bounded and radial $\}$ is dense in $\mathfrak{T}\left(L^{\infty}\right) \cap R a d$.

In [8] Korenblum and Zhu proved that if $a \in L^{\infty}$ is radial and $B_{0}\left(T_{a}\right) \equiv 0$ on $\partial D$ then $T_{a}$ is compact. Several results of this type have appeared in the literature for different types of symbols (see, for instance [9] and [12]). These theorems have being widely generalized by a result of Axler and Zheng [2] asserting that if $S$ is a (several variables) polynomial of Toeplitz operators $T_{a}$, with $a \in L^{\infty}$, and $B_{0}(S) \equiv 0$ on $\partial D$ then $S$ is compact. More recently, in [13] Zorboska has observed that the proof of Korenblum and Zhu can be adapted to generalize the result of [8]. That is, if $S \in \mathfrak{L}\left(L_{a}^{2}\right)$ is radial, $n\left(\lambda_{n}(S)-\lambda_{n+1}(S)\right)$ is bounded, and $B_{0} S \equiv 0$ on $\partial D$ then $S$ is compact. It is a simple calculation to verify that if $a \in L^{\infty}$ is radial, the eigenvalues of $T_{a}$ satisfy the above condition (see the proof of Proposition 4.2 below). In the negative direction, it is known that the radial operator $(S f)(z)=f(-z)$ satisfies $B_{0}(S) \equiv 0$ on $\partial D$, although it is obviously not compact.

Our next result is a straightforward application of Theorem 3.3 that provides another generalization of Korenblum and Zhu's theorem.

Corollary 3.4 Let $S \in \mathfrak{L}\left(L_{a}^{2}\right)$ be a radial operator. Then $S$ is compact if and only if $S \in \mathfrak{T}\left(L^{\infty}\right)$ and $B_{0}(S) \equiv 0$ on $\partial D$.

Proof. If $S$ is compact, a theorem of Coburn [4] asserts that $S \in \mathfrak{T}(C(\bar{D}))$, where $C(\bar{D})$ is the algebra of continuous functions of the closed disk, so $S \in \mathfrak{T}\left(L^{\infty}\right)$. Also, for $z \in D$,

$$
\begin{aligned}
\left|\left(B_{0} S\right)(z)\right| & =\left(1-|z|^{2}\right)^{2}\left|\left\langle S K_{z}^{(0)}, K_{z}^{(0)}\right\rangle\right| \\
& \leq\left\|\left(1-|z|^{2}\right) S K_{z}^{(0)}\right\|\left\|\left(1-|z|^{2}\right) K_{z}^{(0)}\right\| \rightarrow 0
\end{aligned}
$$

as $|z| \rightarrow 1$ because $\left(1-|z|^{2}\right) K_{z}^{(0)}$ has norm 1 for every $z \in D$ and tends weakly to 0 when $|z| \rightarrow 1$. Observe that this argument does not use that $S$ is radial.

Now suppose that $S \in \mathfrak{T}\left(L^{\infty}\right)$ and $B_{0}(S) \equiv 0$ on $\partial D$. By Lemma 4.8 of [10], if $T \in \mathfrak{L}\left(L_{a}^{2}\right)$ is any operator such that $B_{n_{0}}(T) \equiv 0$ on $\partial D$ for some $n_{0} \geq 0$, then $B_{n}(T) \equiv 0$ on $\partial D$ for every $n \geq 0$. Thus, $B_{n}(S) \equiv 0$ on $\partial D$ for every $n \geq 0$. It is well-known that a Toeplitz operator with continuous symbol that identically vanishes on $\partial D$ is compact (see [12], p. 107). Consequently $T_{B_{n}(S)}$ is compact for all $n$, and since by Theorem $3.3, T_{B_{n}(S)} \rightarrow S$, so is $S$. 


\section{An essential commutant versus the Toeplitz algebra}

It is natural to ask whether the inclusion $\mathfrak{T}\left(L^{\infty}\right) \subset \mathfrak{L}\left(L_{a}^{2}\right)$ is proper. In [5] (also [6]), Englis obtained an affirmative answer by considering the essential commutant of $T_{z}$. We recall that the essential commutant of an operator $T \in \mathfrak{L}\left(L_{a}^{2}\right)$ is

$$
C_{e}(T)=\left\{S \in \mathfrak{L}\left(L_{a}^{2}\right): T S-S T \text { is compact }\right\} .
$$

Among other things, he proved

(a) $C_{e}\left(T_{z}\right)=\left\{S \in \mathfrak{L}\left(L_{a}^{2}\right): S-T_{z}^{*} S T_{z}\right.$ is compact $\}$,

(b) $C_{e}\left(T_{z}\right)$ is a $C^{*}$-algebra,

(c) $T_{\phi} \in C_{e}\left(T_{z}\right)$ for every $\phi \in L^{\infty}$.

The proof of (a) is algebraic manipulation from the fact that $I-T_{z} T_{z}^{*}$ and $I-T_{z}^{*} T_{z}$ are compact, (b) is straightforward once (a) is proved, and (c) holds because $T_{\phi}-T_{z}^{*} T_{\phi} T_{z}=$ $T_{\left(1-|z|^{2}\right) \phi}$, which is easily seen to be compact when $\phi \in L^{\infty}$. Observe that (b) and (c) yield

$$
\mathfrak{T}\left(L^{\infty}\right) \subset C_{e}\left(T_{z}\right) \subset \mathfrak{L}\left(L_{a}^{2}\right) .
$$

Since the radial operator $S z^{n}=(-1)^{n} z^{n}$ is not in $C_{e}\left(T_{z}\right)$ (see Proposition 4.2), the second inclusion is proper. But, as Englis noticed, this poses a new problem: to determine whether the first inclusion is proper. With the aid of Theorem 3.3 we will see that this is indeed the case.

Let $\ell^{\infty}$ be the Banach space of bounded complex sequences indexed from $n \geq 0$. Consider the linear subspaces

$$
d_{0}=\left\{\left\{z_{n}\right\} \in \ell^{\infty}:\left(z_{n}-z_{n-1}\right) \rightarrow 0\right\}
$$

and

$$
d_{1}=\left\{\left\{z_{n}\right\} \in \ell^{\infty}: n\left(z_{n}-z_{n-1}\right) \in \ell^{\infty}\right\} .
$$

It is clear that $d_{0}$ is closed in $\ell^{\infty}$ and $d_{1} \subset d_{0}$. Consequently $\bar{d}_{1} \subset d_{0}$, where $\bar{d}_{1}$ denotes the closure of $d_{1}$ in $\ell^{\infty}$. Every convergent sequence is in $\bar{d}_{1}$, but the sequence $a_{n}=(-1)^{n} \log (n+$ $1) /(n+1)$ is not in $d_{1}$. Hence, $d_{1}$ is not closed.

Lemma 4.1 The $\ell^{\infty}$-closure of $d_{1}$ is properly contained in $d_{0}$.

Proof. We shall construct a sequence in $d_{0} \backslash \bar{d}_{1}$. Let $a_{n} \geq 0$ be such that $a_{n} \rightarrow 0$ and $n a_{n} \rightarrow \infty$. We define $\lambda_{n}=\sum_{j=0}^{n} \varepsilon_{j} a_{j}$, where $\varepsilon_{j}=1$ or -1 according to the following rule: 
$\varepsilon_{j}=1$ for $j=0, \ldots, n_{1}$ until $\lambda_{n_{1}-1}<1$ and $\lambda_{n_{1}} \geq 1$. Then $\varepsilon_{j}=-1$ for $j=n_{1}, \ldots, n_{2}$ until $\lambda_{n_{2}-1}>0$ and $\lambda_{n_{2}} \leq 0$. Then $\varepsilon_{j}=1$ again until $\lambda_{n_{3}} \geq 1$ for the first time and repeat the process ad infinitum.

Roughly speaking, we are adding the $a_{n}^{\prime} s$ until we equal or pass 1 to the right, then we rest the next $a_{n}^{\prime} s$ until we equal or pass 0 to the left and so forth. Clearly

$$
\sup _{n}\left|\lambda_{n}\right| \leq 1+\sup _{n}\left|a_{n}\right|<\infty
$$

so $\left\{\lambda_{n}\right\} \in \ell^{\infty}$, and since $\lambda_{n}-\lambda_{n-1}=\varepsilon_{n} a_{n} \rightarrow 0,\left\{\lambda_{n}\right\} \in d_{0}$. Let $0<\varepsilon<1 / 10$ and suppose that there is a sequence $\left\{\beta_{n}\right\} \in d_{1}$ such that $\left\|\left\{\lambda_{n}\right\}-\left\{\beta_{n}\right\}\right\|_{\ell^{\infty}}<\varepsilon$. We will arrive to a contradiction. Since $\left\{\beta_{n}\right\} \in d_{1}$ there is a constant $C>0$ depending only on $\left\{\beta_{n}\right\}$ such that

$$
\beta_{j}-\beta_{j-1} \leq \frac{C}{j} \text { for every } j \geq 1 .
$$

If $n_{k}$ denotes the sequence of integers such that $\lambda_{n_{k}} \leq 0$ then $n_{k}<n_{k+1} \rightarrow \infty$. So, by our hypothesis on $a_{j}$, if $n_{k}$ is big enough then $a_{j}>10 C / j$ for every $j \geq n_{k}$. Taking such $n_{k}$ we obtain

$$
\sum_{j=n_{k}+1}^{n_{k}+n} a_{j}>10 \sum_{j=n_{k}+1}^{n_{k}+n} \frac{C}{j}
$$

for every $n \geq 1$. Since $a_{j} \rightarrow 0$ we can choose $n_{k}$ so big that the additional condition $a_{j}<\varepsilon$ holds for $j \geq n_{k}$. Thus, for $n_{k}$ that big, (4.2) holds and in addition there is some $n=n(\varepsilon)$ with

$$
9 \varepsilon \leq \sum_{j=n_{k}+1}^{n_{k}+n} a_{j}<10 \varepsilon<1 .
$$

Since $a_{n_{k}}<\varepsilon$ and $\lambda_{n_{k}} \leq 0$, by the way in which $\lambda_{n_{k}}$ is defined we have $-\varepsilon \leq \lambda_{n_{k}} \leq 0$. Hence (4.3) implies that

$$
-\varepsilon+9 \varepsilon \leq \lambda_{n_{k}}+\sum_{j=n_{k}+1}^{n_{k}+n} a_{j}=\lambda_{n_{k}+n}<10 \varepsilon
$$

On the other hand, because $\left|\beta_{n_{k}}-\lambda_{n_{k}}\right|<\varepsilon$ and $-\varepsilon \leq \lambda_{n_{k}} \leq 0$ then $\beta_{n_{k}} \leq \varepsilon$. Consequently

$$
\begin{aligned}
\beta_{n_{k}+n} & =\beta_{n_{k}}+\left(\beta_{n_{k}+1}-\beta_{n_{k}}\right)+\cdots+\left(\beta_{n_{k}+n}-\beta_{n_{k}+n-1}\right) \\
& \stackrel{\text { by }}{\leq} \varepsilon+\sum_{j=n_{k}+1} \frac{C}{j}
\end{aligned}
$$




$$
\begin{gathered}
\stackrel{\text { by }}{\stackrel{(4.2)}{\leq}} \varepsilon+\frac{1}{10} \sum_{j=n_{k}+1}^{n_{k}+n} a_{j} \\
\stackrel{\text { by (4.3) }}{\leq} \varepsilon+\frac{1}{10} 10 \varepsilon=2 \varepsilon .
\end{gathered}
$$

Thus $\beta_{n_{k}+n} \leq 2 \varepsilon$, and since (4.4) says that $\lambda_{n_{k}+n} \geq 8 \varepsilon$, we cannot have $\left|\beta_{n_{k}+n}-\lambda_{n_{k}+n}\right|<\varepsilon$, a contradiction.

Proposition 4.2 Let $S \in \mathfrak{L}\left(L_{a}^{2}\right)$ be a radial operator. Then

(1) $S-T_{z}^{*} S T_{z}$ is compact if and only if $\left\{\lambda_{n}(S)\right\} \in d_{0}$.

(2) If $S \in \mathfrak{T}\left(L^{\infty}\right)$ then $\left\{\lambda_{n}(S)\right\} \in \bar{d}_{1}$.

Proof. For (1) observe that

$$
T_{z}^{*} z^{k}= \begin{cases}k /(k+1) z^{k-1} & \text { if } k>0 \\ 0 & \text { if } k=0\end{cases}
$$

yields $\left(S-T_{z}^{*} S T_{z}\right) z^{k}=\lambda_{k}(S) z^{k}-\lambda_{k+1}(S)(k+1) /(k+2) z^{k}$. That is, $S-T_{z}^{*} S T_{z}$ is radial and satisfies

$$
\lambda_{k}\left(S-T_{z}^{*} S T_{z}\right)=\lambda_{k}(S)-\lambda_{k+1}(S)+\frac{\lambda_{k+1}(S)}{k+2} .
$$

The operator $S-T_{z}^{*} S T_{z}$ is compact if and only if the last expression tends to 0, which gives the result. For (2) observe that by Theorem 3.3, it is enough to show that if $b \in L^{\infty}$ is radial, then $\left\{\lambda_{n}\left(T_{b}\right)\right\} \in d_{1}$. Since $b$ is radial, using polar coordinates we see that

$$
\frac{\lambda_{n}\left(T_{b}\right)}{(n+1)}=\left\langle b z^{n}, z^{n}\right\rangle=\int_{0}^{1} b(r) r^{2 n} 2 r d r=\int_{0}^{1} b\left(t^{1 / 2}\right) t^{n} d t .
$$

Thus,

$$
\begin{aligned}
\left|\lambda_{n+1}\left(T_{b}\right)-\lambda_{n}\left(T_{b}\right)\right| & \leq \int_{0}^{1}\left|b\left(t^{1 / 2}\right)\right|\left|(n+2) t^{n+1}-(n+1) t^{n}\right| d t \\
& \leq\|b\|_{\infty} \int_{0}^{1}\left|(n+2) t^{n+1}-(n+1) t^{n}\right| d t \\
& =2\|b\|_{\infty}\left(\frac{n+1}{n+2}\right)^{n+1} \frac{1}{n+2} \leq \frac{\|b\|_{\infty}}{n+2} .
\end{aligned}
$$


This proves (2).

A short comment on the proof of (2) in the above proposition. It is fairly easy to see that $d_{1}$ is a self-adjoint subalgebra of $\ell^{\infty}$, and therefore $\bar{d}_{1}$ is a $C^{*}$-algebra. Since, as showed in the proof, $\left\{\lambda_{n}\left(T_{b}\right)\right\} \in d_{1}$ for every $b \in L_{\text {rad }}^{\infty}(D)$ (the algebra of bounded radial functions), it follows that $\left\{\lambda_{n}(S)\right\} \in \bar{d}_{1}$ for every $S \in \mathfrak{T}\left(L_{\text {rad }}^{\infty}(D)\right)$. That much can be proved without using Theorem 3.3. This means that the only feature of Theorem 3.3 that the proposition really needs is that every radial operator in $\mathfrak{T}\left(L^{\infty}\right)$ belongs to $\mathfrak{T}\left(L_{\text {rad }}^{\infty}(D)\right)$.

Corollary 4.3 The inclusion $\mathfrak{T}\left(L^{\infty}\right) \subset C_{e}\left(T_{z}\right)$ is proper.

Proof. By Lemma 4.1 there is a sequence $\left\{\lambda_{n}\right\} \in d_{0} \backslash \bar{d}_{1}$. Let $S$ be the radial operator with eigenvalues $\lambda_{n}$. By Proposition 4.2 then $S \in C_{e}\left(T_{z}\right) \backslash \mathfrak{T}\left(L^{\infty}\right)$.

The results proven here lead naturally to the following problems,

(1) Is every $\left\{\lambda_{n}\right\} \in \bar{d}_{1}$ the sequence of eigenvalues of a radial operator in $\mathfrak{T}\left(L^{\infty}\right)$ ? More generally, can we give a reasonable characterization of the radial operators in $\mathfrak{T}\left(L^{\infty}\right)$ in terms of their eigenvalues?

(2) Is every $S \in \mathfrak{T}\left(L^{\infty}\right)$ the norm limit of $T_{B_{n}(S)}$ ?

I have no strong feelings about the possible answer to the first question, although my guess is that it is probably negative. I believe that the last question may have an affirmative answer.

Acknowledgements: This work has been supported by the grant SAB2001-0019, from the Secretaría de Estado de Educación y Universidades, Spain.

\section{References}

[1] P. Ahern, M. Flores, and W. Rudin, An invariant volume-mean-value property, J. Funct. Anal. 111 (1993), 380-397.

[2] S. Axler and D. Zheng, Compact operators via the Berezin transform, Indiana Univ. Math. J. 47, No. 2 (1998), 387-400.

[3] F. A. Berezin, Covariant and contravariant symbols of operators, Math. USSR-Izv. 6, (1972), 1117-1151. 
[4] L. A. Coburn, Singular integral operators and Toeplitz operators on odd spheres, Indiana Univ. Math. J. 23 (1973), 433-439.

[5] M. Englis, Toeplitz operators on Bergman-type spaces. Ph.D. Thesis, Prague (1991).

[6] M. Englis, Density of algebras generated by Toeplitz operators on Bergman spaces, Ark. Mat. 30, No. 2 (1992), 227-243.

[7] H. Hedenmalm, B. Korenblum and K. Zhu, Theory of Bergman Spaces, Graduate Texts in Mathematics 199, Springer Verlag, New York 2000.

[8] B. Korenblum and K. Zhu, An application of Tauberian theorems to Toeplitz operators, Journal. Op. Theory 33 (1995), 353-361.

[9] K. Stroethoff, Compact Toeplitz operators on Bergman spaces, Math. Proc. Cambridge Philos. Soc. 124 (1998), 151-160.

[10] D. Suárez, Approximation and symbolic calculus for Toeplitz algebras on the Bergman space, Rev. Mat. Iberoamericana, to appear.

[11] P. Wojtaszczyk, Banach Spaces for Analysts, Cambridge Univ. Press, New York 1991.

[12] K. Zhu, Operator Theory in Function Spaces, Marcel Dekker, New York 1990.

[13] N. Zorboska, The Berezin transform and radial operators, Proc. Amer. Math. Soc. 131, No. 3 (2002), 793-800.

Daniel Suárez

Departament de Matemàtiques

Universitat Autònoma de Barcelona

08193, Bellaterra, Barcelona

Spain

dsuarez@mat.uab.es 